\title{
Trace Identities for the matrix Schrödinger operator on the half line with general boundary conditions *
}

\author{
Ricardo Weder ${ }^{\ddagger}$ \\ Departamento de Física Matemática. \\ Instituto de Investigaciones en Matemáticas Aplicadas y en Sistemas. \\ Universidad Nacional Autónoma de México. \\ Apartado Postal 20-126, México DF 01000, México.
}

\begin{abstract}
We prove Buslaev-Faddeev trace identities for the matrix Schrödinger operator on the half line, with general boundary conditions at the origin, and with selfadjoint matrix potentials.
\end{abstract}

\section{Introduction}

In this paper we study the matrix Schrödinger operator on the half line

$$
H_{A, B} \psi:=-\psi^{\prime \prime}+V(x) \psi, \quad x \in(0, \infty)
$$

'where the prime denotes the derivative with respect to the spatial coordinate $x$. Furthermore, the wavefunction $\psi(x)$ 'will either be an $n \times n$ matrix-valued function or it will be a column vector valued function with $n$ components. As 'it is shown in [1]-9] the general selfadjoint boundary conditions at $x=0$ for the matrix Schrödinger operator (1.1) 'can be formulated in several equivalent way. For our purposes, it is more convenient to use the formulation given in in [6]-9] where we state them in terms of constant $n \times n$ matrices $A$ and $B$ as follows,

$$
\begin{gathered}
-B^{\dagger} \psi(0)+A^{\dagger} \psi^{\prime}(0)=0, \\
-B^{\dagger} A+A^{\dagger} B=0, \\
A^{\dagger} A+B^{\dagger} B>0 .
\end{gathered}
$$

Note that $A^{\dagger} B$ is selfadjoint and the selfadjoint matrix $\left(A^{\dagger} A+B^{\dagger} B\right)$ is positive.

*PACS classification (2010) 02.30.Zz; 03.65.-w; 03.65.Ge; 03.65.Nk. Mathematics Subject Classification (2010): 34L25; 34L40; 81U05; 81 Uxx. Research partially supported by project PAPIIT-DGAPA UNAM IN102215

${ }^{\dagger}$ Fellow, Sistema Nacional de Investigadores.

‡Electronic mail: weder@unam.mx. Home page: http://www.iimas.unam.mx/rweder/rweder.html 
It is clear that the matrices $A, B$ are not uniquely defined. It is always possible to multiply them on the right by an invertible matrix $T$ without affecting (1.2), (1.3) and (1.4). Moreover,

$$
H_{A T, B T}=H_{A, B}
$$

We assume that the potential matrix $V(x)$ is a $n \times n$ selfadjoint matrix-valued function that is in the Faddeev class, namely, $V(x)$ and its first moment are integrable in $(0, \infty)$. That is to say, each entry of the matrix $V$ is Lebesgue measurable on $(0, \infty)$ and

$$
\int_{0}^{\infty}(1+x) d x\|V(x)\|<+\infty
$$

By , $\|V(x)\|$ we denote the norm of $V(x)$ as an operator on $\mathbf{C}^{n}$. Clearly, $V(x)$ satisfies (1.6) if and only this equation holds for each of its entries. Moreover, we assume that the potential is infinitely differentiable on $(0, \infty)$ and that,

$$
\left\|\frac{d^{j}}{d x^{j}} V(x)\right\| \leq C_{j}(1+|x|)^{-\rho-j} \text { for some } \rho \in(1,2], \text { and all } j=0,1,2, \cdots .
$$

Furthermore, we always assume that the matrix potential is selfadjoint ( the dagger means matrix adjoint)

$$
V(x)=V(x)^{\dagger}, \quad x \in(0, \infty)
$$

The main result of our paper is Theorem 3.6 where we prove Buslaev-Faddeev trace identities for the matrix Schrödinger operator (1.1) with the most general boundary conditions (1.2 1.4). These trace identities were first proven in the scalar case by Buslaev and Faddeev in [10 (see also [1] ). For a textbook presentation of these results see Section 6 of Chapter 4 of [12. In these remarkable identities the sum of the absolute value of the eigenvalues, to an even or to an odd power, is expressed in terms of the coefficients of the asymptotic expansion for high energy of the logarithm of the Jost function and of the integral over the absolutely continuous spectrum $(0, \infty)$ of the phase of the Jost function in the even case and of the logarithm of the absolute value of the Jost function in the odd case. The coefficients on the asymptotic expansion of the logarithm of the Jost function depend on the (scalar) potential. These identities give formulae for sums of the absolute value of the eigenvalues to an even or odd power (traces) in terms of properties of the absolutely continuos spectrum encoded in the Jost function. Actually, they link the point spectrum with the absolutely continuous spectrum, or in other words, bound state information with scattering information. In [7] we proved Levinson's theorem for the matrix Schrödinger operator with general boundary conditions, that is a trace identity of order zero.

We prove our trace identities adapting to the matrix Schrödinger operator the classical proof in the scalar case that is given, for example, in Section 6 of Chapter 4 of [12. In our case the Jost function is replaced by the determinant of the Jost matrix. The new technical results that made possible to adapt the classical proof to the matrix case are the precise study of the low-energy behavior of the determinant of the Jost matrix that we obtained in Corollary 6.2 of 
[7] assuming that (1.6) holds, the detailed analysis of the high-energy behavior of the determinant of the Jost matrix that is given in Proposition 7.5 of [7] assuming only that the matrix potential is integrable, i.e., that

$$
\int_{0}^{\infty} d x\|V(x)\|<+\infty
$$

Furthermore, we use to prove Theorem 3.6 the results of Theorems 8.1 and 8.5 of [7] where, in particular, we prove that the eigenvalues of $H_{A, B}$ coincide with the zeros of the determinant of the Jost matrix and that the multiplicity of each eigenvalue is equal to the order of the corresponding zero of the determinant of the Jost matrix. Furthermore, we also use in the proof of Theorem 3.6 the asymptotic expansion for high energy for the logarithm of the determinant of the Jost matrix, that we prove in Section 3 of this paper, assuming that $V(x) \in C^{\infty}((0, \infty))$ and that (1.7) holds.

Currently there is a great deal of interest in the spectral and scattering theory of matrix Schrödinger operators on the half-line with general boundary conditions. For a review of the literature see [6]- $[9]$. They are important, for example, in the quantum mechanical scattering of particles with internal structure and in quantum graphs. The matrix Schrödinger operator on the half line (1.1) is equivalent to a star graph, i.e. to a quantum graph with only one vertex and a finite number of edges of infinite length. Physically it corresponds to $n$ very thin quantum wires connected at the vertex. The boundary conditions (1.2), (1.3) and (1.4) impose relations, at the vertex, between the values of the wave functions, and of its derivatives, at different edges. See for example, [1]- [5] ans [13]- 25]

The paper is organized as follows. In Section 2 we state results from [7, 26], that we need, about the Jost solution, the regular solution, the Jost matrix, and in transformations of the matrices $A, B$ that give the boundary conditions. In Section 3 we prove our trace identities and in Section 4 we give examples that illustrate them.

Along the paper we designate by $\mathbf{C}^{+}$the upper-half complex plane, by $\mathbf{R}$ the real axis, and we let $\overline{\mathbf{C}^{+}}:=\mathbf{C}^{+} \cup \mathbf{R}$. For any $k \in \overline{\mathbf{C}^{+}}$we denote by $k^{*}$ its complex conjugate. As we already mentioned, for any matrix $D$ we designate by $D^{\dagger}$ its adjoint. We denote by $C$ a positive constant that is not required take the same value when it appears in different places.

\section{$2 \quad$ Preliminary results}

In this section we introduce certain results that we need. See [7] and [26]. We always assume that the selfadjoint matrix potential $V$ satisfies at least (1.9). We will use $n \times n$ matrix solutions to the equation

$$
-\psi^{\prime \prime}+V(x) \psi=k^{2} \psi, \quad x \in(0, \infty), k \in \overline{\mathbf{C}^{+}}
$$

Let $F, G$ be any pair of $n \times n$ matrix valued functions defined for $x \in(0, \infty)$. The Wronskian, [F;G] is defined as follows, 


$$
[F ; G]:=F G^{\prime}-F^{\prime} G
$$

Remark that for any two $n \times n$ solutions $\phi(k, x)$ and $\psi(k, x)$ to (2.1), each of the Wronskians $\left[\phi\left(k^{*}, x\right)^{\dagger} ; \psi(k, x)\right]$ and $\left[\phi\left(-k^{*}, x\right)^{\dagger} ; \psi(k, x)\right]$ is independent of $x$.

By $f(k, x)$ we denote the Jost solution to (2.1) that is the $n \times n$ matrix solution that satisfies the following asymptotics for $k \in \overline{\mathbf{C}^{+}} \backslash\{0\}$,

$$
f(k, x)=e^{i k x}\left[I_{n}+o(1 / x)\right], \quad f^{\prime}(k, x)=i k e^{i k x}\left[I_{n}+o(1 / x)\right], \quad x \rightarrow+\infty,
$$

with $I_{n}$ the $n \times n$ identity matrix. For each fixed $x$, (see [7, 26]) $f(k, x)$ and $f^{\prime}(k, x)$ are analytic for $k \in \mathbf{C}^{+}$and continuous for $k \in \overline{\mathbf{C}^{+}}$. Clearly, this asymptotics implies that for each fixed $k \in \mathbf{C}^{+}$, each of the $n$ columns of $f(k, x)$ decays exponentially to zero as $x \rightarrow+\infty$.

Another important solution to (2.1) is the regular solution, $\varphi_{A, B}(k, x)$, that is the $n \times n$ matrix solution defined by the the initial conditions

$$
\varphi_{A, B}(k, 0)=A, \quad \varphi_{A, B}^{\prime}(k, 0)=B
$$

with $A$ and $B$ the matrices that define the boundary conditions in (1.2), (1.3), (1.4). $\varphi_{A, B}(k, x)$ is entire in $k$ in the complex plane $\mathbf{C}$, for each fixed $x \in(0, \infty)$.

Let us define the Jost matrix $J(k)$ in the following way,

$$
J_{A, B}(k):=\left[f\left(-k^{*}, x\right)^{\dagger} ; \varphi_{A, B}(k, x)\right], \quad k \in \overline{\mathbf{C}^{+}} .
$$

It follows from (2.4) and evaluating the Wronskian at $x=0$, that,

$$
J_{A, B}(k)=f\left(-k^{*}, 0\right)^{\dagger} B-f^{\prime}\left(-k^{*}, 0\right)^{\dagger} A, \quad k \in \overline{\mathbf{C}^{+}} .
$$

$J(k)$ is well defined for $\overline{\mathbf{C}^{+}}$since $f\left(-k^{*}, 0\right)^{\dagger}$ and $f^{\prime}\left(-k^{*}, 0\right)^{\dagger}$ are analytic in $k \in \mathbf{C}^{+}$and continuous in $k \in \overline{\mathbf{C}^{+}}$. It is known [7] that $J(k)$ is invertible for $k \in \mathbf{R} \backslash 0$.

It is proven in Proposition 4.1 of [7] (with $M, M^{\dagger}$ there, replaced, respectively, by $M^{\dagger}, M$ ) that under the unitary transformation $V \mapsto M V M^{\dagger}$, with a unitary matrix $M$, and the combination of three consecutive transformations $(A, B) \mapsto\left(M A T_{1} M^{\dagger} T_{2}, M B T_{1} M^{\dagger} T_{2}\right)$, first by a right multiplication by an invertible matrix $T_{1}$, then by the unitary 
transformation with $M$, followed by a right multiplication by an invertible matrix $T_{2}$, we have that

$$
\begin{aligned}
f_{M V M^{\dagger}}(k, x) & =M f(k, x) M^{\dagger}, \quad k \in \overline{\mathbf{C}^{+}}, \\
\varphi_{M V M^{\dagger}, M A T_{1} M^{\dagger} T_{2}, M B T_{1} M^{\dagger} T_{2}}(k, x) & =M \varphi_{V, A, B}(k, x) T_{1} M^{\dagger} T_{2}, \quad k \in \mathbf{C}, \\
J_{M V M^{\dagger}, M A T_{1} M^{\dagger} T_{2}, M B T_{1} M^{\dagger} T_{2}}(k, x) & =M J_{V, A, B}(k, x) T_{1} M^{\dagger} T_{2}, \quad k \in \overline{\mathbf{C}^{+}} .
\end{aligned}
$$

Furthermore, we have that,

$$
H_{M V M^{\dagger}, M A T_{1} M^{\dagger} T_{2}, M B T_{1} M^{\dagger} T_{2}}=M H_{V, A, B} M^{\dagger}
$$

For clarity, in (2.6)-(2.9) we explicitly state the dependence in $V$ of the Jost solution, the regular solution, the Jost matrix and the matrix Schrödinger operator.

The transformation $V \mapsto V$ and $(A, B) \mapsto(A T, B T)$ with an invertible matrix $T$ is just a change in the parametrization of the boundary conditions (1.2), (1.3) and (1.4). On the contrary, the unitary transformation $V \mapsto M V M^{\dagger}$ and $(A, B) \mapsto\left(M A M^{\dagger}, M B M^{\dagger}\right)$ with a unitary matrix $M$ is a change of representation in quantum mechanical sense.

Taking into account the general selfadjoint boundary condition for the scalar Schrödinger operator, that is to say when $n=1$, we consider the case where the matrices $A, B$ are diagonal. This special pair of diagonal matrices is denoted by $\tilde{A}$ and $\tilde{B}$, with,

$$
\tilde{A}:=-\operatorname{diag}\left\{\sin \theta_{1}, \ldots, \sin \theta_{n}\right\}, \quad \tilde{B}:=\operatorname{diag}\left\{\cos \theta_{1}, \ldots, \cos \theta_{n}\right\}
$$

In this case the boundary conditions (1.2) are,

$$
\cos \theta_{j} \psi_{j}(0)+\sin \theta_{j} \psi_{j}^{\prime}(0)=0, \quad j=1,2, \cdots, n
$$

Here, the real parameters $\theta_{j}$ take values in the interval $(0, \pi]$. The case $\theta_{j}=\pi$ corresponds to the Dirichlet boundary condition, the case where $\theta_{j} \neq \pi / 2, \pi$ corresponds to mixed boundary conditions and the case $\theta_{j}=\pi / 2$ corresponds to the Neumann boundary condition. We assume that there $n_{\mathrm{D}}$ values with $\theta_{j}=\pi$, that there are $n_{\mathrm{M}}$ values $\theta_{j} \neq \pi / 2, \pi$ and, in consequence, that there are $n_{\mathrm{N}}$ remaining values, with $n_{\mathrm{N}}:=n-n_{\mathrm{M}}-n_{\mathrm{D}}$, such that the corresponding $\theta_{j}$-values are $\theta_{j}=\pi / 2$. Particular cases where any of $n_{\mathrm{D}}, n_{\mathrm{M}}$, and $n_{\mathrm{N}}$ are zero or $n$ are allowed. Clearly, $\tilde{A}, \tilde{B}$ satisfy (1.3), (1.4) with $\tilde{A}, \tilde{B}$ instead of $A, B$.

In Proposition 4.3 of [7] it is proven that for any pair of matrices $(A, B)$ that satisfy (1.2)-(1.4) there is a pair of diagonal matrices $(\tilde{A}, \tilde{B})$ as in (2.10), a unitary matrix $M$ and a two invertible matrices $T_{1}, T_{2}$ such that,

$$
A=M \tilde{A} T_{2} M^{\dagger} T_{1}, \quad B=M \tilde{B} T_{2} M^{\dagger} T_{1}
$$

Note that $T_{1}, T_{2}$ in (2.12) correspond, respectively to $T_{1}^{-1}, T_{2}^{-1}$ in Proposition 4.3 of 7 . 
The following results are proven in Proposition 6.1 of [7]. Consider the selfadjoint matrix Schrödinger operator (1.1) with the selfadjoint boundary conditions (1.2, 1.3, 1.4) and with the selfadjoint potential $V$ in the Faddeev class (1.6), Then, the nonzero column vector $u \in \mathbf{C}^{n}$ is an eigenvector of the zero-energy Jost matrix $J(0)$ with the zero eigenvalue, i.e. $u \in \operatorname{Ker}[J(0)]$ if and only if $\varphi_{A, B}(0, x) u$ is bounded for $x \in(0, \infty)$. Let us denote by $\mu$ the geometric multiplicity of the zero eigenvalue of $J(0)$. Then, we can form exactly $\mu$ columns by using linear combinations of $n$ columns of the zero-energy regular solution $\varphi_{A, B}(0, x)$ in such a way that those $\mu$ columns form linearly independent solutions to the zero-energy Schrödinger equation,

$$
-\varphi^{\prime \prime}(x)+V(x) \varphi(x)=0,
$$

that satisfy the boundary conditions $(1.2,1.3,1.4)$ and that remain bounded as $x \rightarrow+\infty$. Furthermore, each of such $\mu$ column-vector solutions to (2.13) can also be expressed as a linear combinations of columns of $f(0, x)$. In the physics literature the bounded solutions to (2.13) that satisfy the boundary conditions are called half-bound states or zero energy resonances. Hence, $\mu$ is the number of linearly independent half-bound states or of zero energy resonances.

Furthermore, in Corollary 6.2 of [7] it is proven that if the selfadjoint potential is in the Fadeev class (1.6), the determinant of the Jost matrix $J(k)$ defined in (2.4) has the small- $k$ behavior

$$
\operatorname{det} J(k)=c_{1} k^{\mu}[1+o(1)], \quad k \rightarrow 0 \text { in } \overline{\mathbf{C}^{+}}
$$

where $c_{1}$ is a nonzero constant that it is explicitly given in [7].

Furthermore, it is proven in Proposition 7.5 of $[7$ that if the selfadjoint potential is integrable, i.e. if it satisfies (1.9), then,

$$
\operatorname{det} J(k)=c_{2} k^{n_{M}+n_{N}}[1+O(1 / k)], \quad k \rightarrow \infty \text { in } \overline{\mathbf{C}^{+}},
$$

where $c_{2}$ is a nonzero constant that it is explicitly given in [7, and $n_{M}$ and $n_{N}$ are the nonnegative integers defined after (2.11), i.e. they are, respectively, the number of mixed and of Neumann boundary conditions in the representation where the matrices that define the boundary conditions are given by the diagonal matrices $\tilde{A}, \tilde{B}$.

The following results concerning eigenvalues of $H_{A, B}$ are proven in [7. Since the matrix Schrödinger operator, $H_{A, B}$ is selfadjoint its eigenvalues, $k^{2}$, have to be real. It turns out that $H_{A, B}$ has no eigenvalues for $k^{2} \geq 0$ (see the beginning of Section VIII of [7]). Then, the eigenvalues appear at $k^{2}$, for $k=i \kappa$, for some $\kappa>0$.

It is proven in Theorem 8.1 of [7] that we have an eigenvalue at $k=i \kappa$ for some positive $\kappa$ if and only if $\operatorname{Ker}[J(i \kappa)]$ is nontrivial or equivalently if and only if $\operatorname{det}[J(i \kappa)]=0$. The multiplicity, $m_{\kappa}$, of the eigenvalue at $k=i \kappa$ is finite and it is equal to the dimension of $\operatorname{Ker}[J(i \kappa)]$ Furthermore, it is proven in Theorem 8.5 of [7] that, 


$$
\operatorname{det} J(k)=c_{3}(k-i \kappa)^{m_{\kappa}}[1+O(k-i \kappa)], \quad k \rightarrow i \kappa,
$$

where $c_{3}$ is a nonzero constant. Consequently, the order of the zero of $\operatorname{det} J(k)$ at $k=i \kappa$ is equal to the multiplicity, $m_{\kappa}$, of the eigenvalue at $k=i \kappa$. We denote by $\mathcal{N}$ the total number of eigenvalues of $H_{A, B}$, repeated according to its multiplicity. If (1.6) holds, $\mathcal{N}<\infty$.

\section{$3 \quad$ Trace Identities}

We first obtain a high-energy asymptotic expansion for the Jost solution. Let us denote,

$$
m(k, x):=e^{-i k x} f(k, x)
$$

LEMMA 3.1. Suppose that the matrix potential $V$ is selfadjoint, that it belongs to $C^{\infty}((0, \infty))$ and that it satisfies (1.7). Then, for any $N=0,1,2, \cdots$,

$$
m(k, x)=\sum_{l=0}^{N} \frac{1}{(2 i k)^{l}} b_{l}(x)+r_{N}(k, x),
$$

where the remainder $r_{N}$ satisfies the estimate: for every $C>0$ there are constants $C_{N, j}$ such that,

$$
\left|\frac{\partial^{j}}{\partial x^{j}} r_{N}(k, x)\right| \leq C_{N, j} \frac{1}{|k|^{N+1}} \frac{1}{(1+|x|)^{(N+1)(\rho-1)+j}}, \quad j=0,1,2, \cdots, x \in[0, \infty), \operatorname{Im} k \geq 0,|k| \geq C,
$$

where $b_{l}(x), l=0,1,2, \cdots$ are $C^{\infty}$ functions defined by the following recurrent relation,

$$
b_{0}(k, x)=1, \quad b_{l+1}(x)=-b_{l}^{\prime}(x)-\int_{x}^{\infty} V(y) b_{l}(y) d y .
$$

The $b_{l}(x)$ satisfy the estimate,

$$
\left|\frac{d^{j}}{d x^{j}} b_{l}(x)\right| \leq C_{l, j} \frac{1}{|x|^{l(\rho-1)+j}}, \quad j=0,1,2, \cdots, l=0,1,2, \cdots .
$$

Furthermore the expansion (3.2) can be derived term by term.

REMARK 3.2. When an asymptotic expansion as (3.2) holds for all $N$ we will write the right-hand side as an asymptotic series (see [27])

$$
m(k, x)=\sum_{l=0}^{\infty} \frac{1}{(2 i k)^{j}} b_{l}(x) .
$$

Proof: Results of this type are classical. For the reader's convenience we outline the proof following the one given in the scalar case in Proposition 4.1 in Chapter 4 of [12, with some changes. The coefficients $b_{l}, l \geq 1$ are defined by the 
recurrent formula (3.4) and one easily checks that (3.5) is satisfied. Inserting (3.1) and (3.2) into (2.1) we obtain the equation for the remainder,

$$
-r_{N}^{\prime \prime}(k, x)-2 i k r_{N}^{\prime}(k, x)+V(x) r_{N}(k, x)=q_{N}(k, x)
$$

where,

$$
q_{N}(k, x):=\left(b_{N}^{\prime \prime}(x)-V(x) b_{N}(x)\right) \frac{1}{(2 i k)^{N}} .
$$

By 1.7. 3.5

$$
\left|\frac{\partial^{j}}{\partial x^{j}} q_{N}(k, x)\right| \leq C_{N, j}(1+x)^{-(N+1)(\rho-1)-1-j} \frac{1}{(2 i k)^{N}} .
$$

Writing (3.7) as an integral equation we get,

$$
r_{N}(k, x)=Q_{N}(k, x)+\frac{1}{2 i k} \int_{x}^{\infty}\left(e^{2 i k(y-x)}-1\right) V(y) r_{N}(k, y) d y,
$$

where,

$$
Q_{N}(k, x):=\frac{1}{2 i k} \int_{x}^{\infty}\left(1-e^{2 i k(y-x)}\right) q_{N}(k, y) d y
$$

By (3.9)

$$
\left|Q_{N}(k, x)\right| \leq C_{N}(1+x)^{-(N+1)(\rho-1)} \frac{1}{(2 i k)^{N+1}} .
$$

Then, solving (3.10) by iterations and using (3.12) we prove that $r_{N}(k, x)$ satisfies (3.3) with $j=0$. To prove it for positive $j$ we proceed as follows (in the proof of Proposition 4.1 in Chapter 4 of [12] in the scalar case a different argument is proposed). We assume that (3.3) is true for $j=0,1, \cdots j_{0}$ and we prove it for $j=j_{0}+1$. We denote $p(k, x):=\frac{\partial^{j_{0}+1}}{\partial x^{j_{0}+1}} r_{N}(k, x)$. Then, deriving (3.7) $j_{0}+1$ times we get that,

$$
-p^{\prime \prime}(k, x)-2 i k p^{\prime}(k, x)+V(x) p(k, x)=s(k, x),
$$

where,

$$
s(k, x)=-\sum_{j=1}^{j=j_{0}+1}\left(\begin{array}{c}
j_{0}+1 \\
j
\end{array}\right)\left(\frac{d x^{j}}{d x^{j}} V(x)\right) \frac{\partial^{j_{0}+1-j}}{\partial x^{j_{0}+1-j}} r_{N}(k, x)+\frac{\partial^{j_{0}+1}}{\partial x^{j_{0}+1}} q_{N}(k, x) .
$$

By (1.7), (3.9) and the inductive assumption we have that,

$$
|s(k, x)| \leq C(1+x)^{-(N+1)(\rho-1)-j_{0}-2} \frac{1}{(2 i k)^{N}} .
$$

Writing (3.13) as an integral equation we obtain that,

$$
p(k, x)=S(k, x)+\frac{1}{2 i k} \int_{x}^{\infty}\left(e^{2 i k(y-x)}-1\right) V(y) p(k, y) d y
$$

where,

$$
S(k, x):=\frac{1}{2 i k} \int_{x}^{\infty}\left(1-e^{2 i k(y-x)}\right) s(k, y) d y
$$


By (3.15) we have that $S(k, x)$ satisfies

$$
|S(k, x)| \leq(1+x)^{-(N+1)(\rho-1)-j_{0}-1} \frac{1}{(2 i k)^{N+1}} .
$$

Finally, solving (3.16) by iterations and using (3.18) we obtain that,

$$
|p(k, x)| \leq(1+x)^{-(N+1)(\rho-1)-j_{0}-1} \frac{1}{(2 i k)^{N+1}},
$$

what proves that (3.3) holds for $r_{N}(k, x)$ with $j=j_{0}+1$.

By Lemma 3.1 the Jost matrix $J(k)$ defined in (2.4) (see also (2.5) ) has the following asymptotic expansion,

$$
J(k)=f\left(-k^{*}, 0\right)^{\dagger} B-f^{\prime}\left(-k^{*}, 0\right)^{\dagger} A=\sum_{l=-1}^{\infty} c_{l} \frac{1}{(2 i k)^{l}},
$$

where,

$$
\begin{aligned}
c_{-1} & =-\frac{1}{2} A, \\
c_{0} & =B-\frac{1}{2} b_{1}(0)^{\dagger} A, \\
c_{l} & \left.=b_{l}(0)^{\dagger} B-\left(\frac{1}{2} b_{l+1}(0)^{\dagger}\right]+b^{\prime}(0)_{l}^{\dagger}\right) A, l=1,2, \cdots .
\end{aligned}
$$

Let us denote by $\mathcal{P}$ the set of all permutations of $1,2, \cdots, n$. By (3.20) we have that

$$
\operatorname{det} J(k)=\sum_{l=-n}^{\infty} d_{l} \frac{1}{(2 i k)^{l}},
$$

with

$$
d_{l}:=\sum_{\sigma \in \mathcal{P}} \operatorname{sign} \sigma \sum_{l_{i} \geq-1: l_{1}+l_{2}+\cdots l_{n}=l}\left(c_{l_{1}}\right)_{1, \sigma_{1}}\left(c_{l_{2}}\right)_{2,, \sigma_{2}} \cdots\left(c_{l_{n}}\right)_{n, \sigma_{n}},
$$

where by $\left(c_{l}\right)_{j, m}$ we denote the component of the matrix $c_{l}$ in the row $j$ and the column $m$.

By (2.15),

$$
d_{j}=0 \text { if } j<-n_{M}-n_{N}, \quad \text { and } d_{-n_{M}-n_{N}}=\frac{c_{2}}{(2 i)^{n_{M}+n_{N}}} .
$$

Then, we have proven the following lemma.

LEMMA 3.3. Suppose that the matrix potential $V$ is selfadjoint, that it belongs to $C^{\infty}((0, \infty))$ and that it satisfies (1.7). Then, the determinant of the Jost matrix, defined in (2.4), has the following asymptotic expansion

$$
\operatorname{det} J(k)=\sum_{l=-n_{M}-n_{N}}^{\infty} d_{l} \frac{1}{(2 i k)^{l}},
$$

where the coefficients $d_{l}, l=-n_{M}-n_{N},-n_{M}-n_{N}+1, \cdots$ are defined in 3.4, 3.21, 3.23, 3.24) with $c_{2}$ the constant that appears in (2.15) and $n_{M}, n_{N}$ the nonnegative integers defined after equation (2.11). 
Let us denote,

$$
h(k):=\frac{1}{c_{2} k^{n_{M}+n_{N}}} \operatorname{det} J(k) .
$$

By (2.15), $\lim _{|k| \rightarrow \infty} h(k)=1$. Hence, we define $\ln h(k)$ with $\lim _{|k| \rightarrow \infty} \ln h(k)=0$. By (3.25)

$$
\ln h(k)=\sum_{l=1}^{\infty} e_{l} \frac{1}{(2 i k)^{l}},
$$

where,

$$
\begin{aligned}
e_{1} & =\frac{(2 i)^{n_{M}+n_{N}}}{c_{2}} d_{1-n_{M}-n_{N}}, \\
e_{l} & =\frac{(2 i)^{n_{M}+n_{N}}}{c_{2}}\left(d_{l-n_{M}-n_{N}}-\frac{1}{l} \sum_{j=1}^{l-1} j d_{l-n_{M}-n_{N}-j} e_{j}\right), l=2, \cdots .
\end{aligned}
$$

For $k$ real we split the asymptotic expansion (3.27) into its even and odd parts,

$$
\ln h(k)=\ln _{e} h(k)+\ln _{o}(k), \ln _{e} h(k):=\frac{1}{2}(\ln h(k)+\ln h(-k)), \quad \ln _{o} h(k):=\frac{1}{2}(\ln h(k)-\ln h(-k)), \quad k \in \mathbf{R} .
$$

By (3.27),

$$
\begin{gathered}
\ln _{e} h(k)=\sum_{l=1}^{\infty} e_{2 l} \frac{1}{(2 i k)^{2 l}}=\sum_{l=1}^{\infty}(-1)^{l} e_{2 l} \frac{1}{(2 k)^{2 l}}, \quad k \in \mathbf{R}, \\
\ln _{o} h(k):=\sum_{l=0}^{\infty} e_{2 l+1} \frac{1}{(2 i k)^{2 l+1}}=i \sum_{l=0}^{\infty}(-1)^{l+1} e_{2 l+1} \frac{1}{(2 k)^{2 l+1}}, \quad k \in \mathbf{R} .
\end{gathered}
$$

LEMMA 3.4. Suppose that the selfadjoint matrix potential $V$ satisfies (1.6). For any $z \in \mathbf{C}$ with $0<\operatorname{Re} z<1 / 2$ let us define,

$$
F(z):=\int_{0}^{\infty} \ln _{e} h(k) k^{2 z-1} d z, \quad G(z):=-i \int_{0}^{\infty} \ln _{o} h(k) k^{2 z-1} d z .
$$

Then,

$$
F(z) \sin (\pi z)-G(z) \cos (\pi z)=\frac{\pi}{2 z} \widetilde{\sum_{j=1}^{\mathcal{N}}}\left|\kappa_{j}\right|^{2 z}
$$

where $-\kappa_{j}^{2}, j=1,2 \cdots, \mathcal{N}$ are the eigenvalues of the Schrödinger operator $H_{A, B}$ defined in (1.1) with the boundary conditions [1.2- 1.4]. In the right-hand side of (3.33) $\widetilde{\Sigma}_{j=1}^{\mathcal{N}}\left|\kappa_{j}\right|^{2 z}$ means the sum over the absolute value of the eigenvalues, $-\kappa_{j}^{2}$, to the power $z$ and repeated according to its multiplicity. Furthermore, $\mathcal{N}$ is the total number of (repeated) eigenvalues. As (1.6) holds, $\mathcal{N}<\infty$.

Proof: This lemma is proven as in the proof of Proposition 6.3 in Chapter 4 of 12 integrating the function $(\dot{h}(k) / h(k)) k^{2 z}$, with $h(k)$ defined in (3.26) with $0 \leq \arg k \leq \pi$ along the contour $\mathcal{C}_{\epsilon, R}$, given below, taking the limit when $\epsilon \rightarrow 0$ and $R \rightarrow \infty$ and using (2.14), (2.15), (2.16) and the residues theorem. The contour $\mathcal{C}_{\epsilon, R}$ consists of four parts given by

$$
\mathcal{C}_{\epsilon, R}:=(-R,-\epsilon) \cup \mathcal{C}_{\epsilon} \cup(\epsilon, R) \cup \mathcal{C}_{R}
$$


The piece $(-R,-\epsilon)$ is the directed line segment on the real axis for some small positive $\epsilon$ and for a large positive $R$, with the direction of the path from $-R+i 0$ to $-\epsilon+i 0$. The second part $\mathcal{C}_{\epsilon}$ consists of the upper semicircle centered at the origin with radius $\epsilon$ and traversed from the point $-\epsilon+i 0$ to the point $\epsilon+i 0$. The third piece $(\epsilon, R)$ is the directed line segment of the positive real axis from $\epsilon+i 0$ to $R+i 0$. The fourth part $\mathcal{C}_{R}$ is the upper semicircle centered at the origin with radius $R$ and traversed from the point $R+i 0$ to the point $-R+i 0$.

PROPOSITION 3.5. Assume that the selfadjoint matrix potential $V$ satisfies (1.6), that it belongs to $C^{\infty}((0, \infty))$ and that (1.7) holds. Then, the functions $F(z)$ and $G(z)$, defined in (3.32) have analytic continuations, denoted also by $F(z), G(z)$, to meromorphic functions for $\operatorname{Re} z>0$. The function $F(z)$ has simple poles at $z=j, j=1,2, \cdots$ with residue $(-1)^{j+1} 2^{-2 j-1} e_{2 j}$. Furthermore, the representation (3.32) of $F(z)$ is valid for $0<\operatorname{Re} z<1$, and,

$$
F(z)=\int_{0}^{\infty}\left(\ln _{e} h(k)-\sum_{l=1}^{j}(-1)^{l} e_{2 l} \frac{1}{(2 k)^{2 l}}\right) k^{2 z-1} d k, \quad \text { for } j<\operatorname{Re} z<j+1, j=1,2, \cdots
$$

Moreover, $G(z)$ has simple poles at $z=j+1 / 2, j=0,1,2, \cdots$ with residue, $(-1)^{j} 2^{-2 j-2} e_{2 j+1}$ and

$$
G(z)=\int_{0}^{\infty}\left(-i \ln _{o} h(k)-\sum_{l=0}^{j-1}(-1)^{l+1} e_{2 l+1} \frac{1}{(2 k)^{2 l+1}}\right) k^{2 z-1} d k, \quad j-\frac{1}{2}<\operatorname{Re} z<j+\frac{1}{2}, j=1,2, \cdots
$$

Proof: The proposition follows from (3.30), (3.31) as in the proof of Lemma 6.4 in Chapter 4 of [12. We give details in the case of $G(z)$ for the reader's convenience. We have that for Rez $<\frac{1}{2}$,

$$
\begin{array}{r}
G(z)=\int_{0}^{1}(-i) \ln _{o} h(k) k^{2 z-1} d k+\int_{1}^{\infty}\left(-i \ln _{o} h(k)-\sum_{l=0}^{j-1} \frac{(-1)^{l+1} e_{2 l+1}}{(2 k)^{2 l+1}}\right) k^{2 z-1} d k \\
+\sum_{l=0}^{j-1}(-1)^{l} 2^{-2 l-2} \frac{e_{2 l+1}}{z-l-1 / 2} .
\end{array}
$$

The first integral in the right-hand side of (3.36) is analytic for Rez $>0$ and by (3.31) the second integral is analytic for $\operatorname{Re} z<j+1 / 2$. Since this is true for every $j=1,2, \cdots, G(z)$ has an analytic continuation to a meromorphic function for $\operatorname{Re} z>0$ with simple poles at $z=j+1 / 2, j=0,1,2, \cdots$, with residue, $(-1)^{j} 2^{-2 j-2} e_{2 j+1}$. Moreover, for $j-\frac{1}{2}<\operatorname{Re} z$

$$
\begin{array}{r}
\int_{0}^{1}(-i) \ln _{o} h(k) k^{2 z-1} d k=\int_{0}^{1}\left(-i \ln _{o} h(k)-\sum_{l=0}^{j-1} \frac{(-1)^{l+1} e_{2 l+1}}{(2 k)^{2 l+1}}\right) k^{2 z-1} d k \\
-\sum_{l=0}^{j-1}(-1)^{l} 2^{-2 l-2} e_{2 l+1} \frac{1}{z-l-1 / 2} .
\end{array}
$$

Equation (3.35) follows from (3.36) and (3.37).

We now prove our main result. 
THEOREM 3.6. Suppose that the selfadjoint matrix potential $V$ satisfies (1.6) that it belongs to $C^{\infty}((0, \infty))$ and that (1.7) holds. Then,

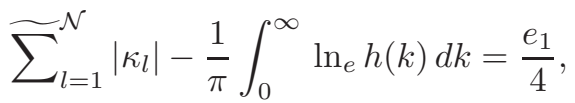

$$
\begin{aligned}
& {\widetilde{\sum_{l=1}^{\mathcal{N}}}}_{\left.\kappa_{l}\right|^{2 j+1}+(-1)^{j+1}} \frac{2 j+1}{\pi} \int_{0}^{\infty}\left(\ln _{e} h(k)-\sum_{l=1}^{j}(-1)^{l} e_{2 l} \frac{1}{(2 k)^{2 l}}\right) k^{2 j} d k=\frac{(2 j+1) e_{2 j+1}}{2^{2 j+2}}, j=1,2, \cdots .
\end{aligned}
$$

Furthermore,

$$
\widetilde{\sum_{l=1}^{\mathcal{N}}}\left|\kappa_{l}\right|^{2 j}+(-1)^{j} \frac{2 j}{\pi} \int_{0}^{\infty}\left(-i \ln _{o} h(k)-\sum_{l=0}^{j-1}(-1)^{l+1} e_{2 l+1} \frac{1}{(2 k)^{2 l+1}}\right) k^{2 j-1} d k=-j \frac{e_{2 j}}{2^{2 j}}, j=1,2, \cdots .
$$

In the left -hand side of (3.38), (3.39) and (3.40) $\widetilde{\sum}_{l=1}^{\mathcal{N}}\left|\kappa_{l}\right|^{q}$, with, respectively, $q=1, q=2 j+1$, and $q=2 j$, means the sum over the absolute value of the eigenvalues, $-\kappa_{l}^{2}$, to the power $q / 2$, and repeated according to its multiplicity. Furthermore, $\mathcal{N}$ is the total number of (repeated) eigenvalues. As (1.6) holds, $\mathcal{N}<\infty$. The coeficients $e_{j}, j=1,2, \cdots$ are defined in equations 3.4, 3.21, 3.23, 3.24, 3.28).

Proof: By Proposition 3.5 and analytic continuation (3.33) holds for $\operatorname{Re} z>0$. Equations (3.38) and (3.39) follow evaluating (3.33) at $z=j+1 / 2, j=0,1,2, \cdots$ and using (3.34). Moreover, (3.40) follows evaluating (3.33) at $z=j, j=1,2, \cdots$ and using (3.35).

\section{Examples}

In this section we illustrate our trace identities in Theorem 3.6 with simple examples.

EXAMPLE 4.1. We consider a $2 \times 2$ system, i.e. $n=2$, with Dirichlet boundary condition, $\psi(0)=0$. We can take, $A=0, B=-I$. Since we only have Dirichlet boundary conditions according to the definition given below equation (2.11) $n_{M}=n_{N}=0$ and also the constant $c_{2}$ that appears in (2.15) is equal to one (see the first equation in page 16 of [7]). Then according to (3.4, 3.21, 3.23, 3.24, 3.28).

$$
\begin{gathered}
e_{1}=-\int_{0}^{\infty} V_{1,1}(x) d x-\int_{0}^{\infty} V_{2,2}(x) d x \\
e_{2}=\left(\int_{0}^{\infty} V_{1,1}(x) d x\right)\left(\int_{0}^{\infty} V_{2,2}(x) d x\right)-\left(\int_{0}^{\infty} V_{1,2}(x) d x\right)\left(\int_{0}^{\infty} V_{2,1}(x) d x\right)-V_{1,1}(0) \\
+\left(\int_{0}^{\infty} d x \int_{x}^{\infty} V(y) d y V(x)\right)_{1,1}-V_{2,2}(0)+\left(\int_{0}^{\infty} d x \int_{x}^{\infty} V(y) d y V(x)\right)_{2,2} \\
-\frac{1}{2}\left(\int_{0}^{\infty} V_{1,1}(x) d x+\int_{0}^{\infty} V_{2,2}(x) d x\right)^{2} .
\end{gathered}
$$


EXAMPLE 4.2. (The $\delta^{\prime}$ boundary condition). We consider a $3 \times 3$ system i.e., $n=3$. It satisfies the $\delta^{\prime}$ boundary condition,

$$
\psi_{1}^{\prime}(0)=\psi_{2}^{\prime}(0)=\psi_{3}^{\prime}(0), \quad \sum_{j=1}^{3} \psi_{j}(0)=a \psi_{1}^{\prime}(0), a \in \mathbf{R} .
$$

The matrices $A, B$ can be taken as,

$$
A=\left[\begin{array}{ccc}
1 & 0 & -a \\
-1 & 1 & 0 \\
0 & -1 & 0
\end{array}\right], \quad B=\left[\begin{array}{ccc}
0 & 0 & -1 \\
0 & 0 & -1 \\
0 & 0 & -1
\end{array}\right]
$$

The potential $V$ is identically zero. The matrices $A, B$ satisfy (1.3), (1.4). From (2.5) with $f(k, x)=e^{i k x} I_{3}$, we obtain that,

$$
J_{A, B}(k)=\left[\begin{array}{ccc}
-i k & 0 & -1+i a k \\
i k & -i k & -1 \\
0 & i k & -1
\end{array}\right]
$$

Then,

$$
\operatorname{det} J_{A, B}(k)=k^{2}(3-i a k)
$$

It follows that if $a \geq 0$ the Schrödinger operator $H_{A, B}$ has no eigenvalues. On the contrary, if $a<0, H_{A, B}$ has one eigenvalue $-\kappa_{1}^{2}$ with $i \kappa_{1}=i 3 /|a|$ and with multiplicity one. Suppose that $a \neq 0$. Then, with $h(k)$ defined in (3.26)

$$
h(k)=\frac{\operatorname{det} J_{A, B}(k)}{-i a k^{3}}=\left(1+\frac{3 i}{a k}\right) .
$$

Then,

$$
\ln h(k)=\sum_{l=1}^{\infty} \frac{-1}{l}\left(\frac{6}{a}\right)^{l} \frac{1}{(2 i k)^{l}}
$$

It follows that

$$
e_{l}=\frac{-1}{l}\left(\frac{6}{a}\right)^{l}, l=1,2, \cdots .
$$

In the case $a=0, h(k)=1$ and then $\ln h(k)=0$ and $e_{l}=0, l=1,2,3, \cdots$.

\section{References}

[1] V. Kostrykin and R. Schrader, Kirchhoff's rule for quantum wires, J. Phys. A 32, 595-630 (1999).

[2] V. Kostrykin and R. Schrader, Kirchhoff's rule for quantum wires. II: The inverse problem with possible applications to quantum computers, Fortschr. Phys. 48, 703-716 (2000).

[3] M. S. Harmer, Inverse scattering for the matrix Schrödinger operator and Schrödinger operator on graphs with general self-adjoint boundary conditions, ANZIAM J. 44, 161-168 (2002).

[4] M. S. Harmer, The matrix Schrödinger Operator and Schrödinger Operator on Graphs, Ph.D. thesis, University of Auckland, New Zealand, 2004. 
[5] M. S. Harmer, Inverse scattering on matrices with boundary conditions, J. Phys. A 38, 4875-4885 (2005).

[6] T. Aktosun, M. Klaus, and R. Weder, Small-energy analysis for the self-adjoint matrix Schrödinger equation on the half line, J. Math. Phys. 52, 102101 (2011).

[7] T. Aktosun, and R. Weder, High-energy analysis and Levinson's theorem for the self-adjoint matrix Schrödinger operator on the half line, J. Math. Phys. 54, 012108 (2013).

[8] T. Aktosun, M. Klaus, and R. Weder, Small-energy analysis for the self-adjoint matrix Schrödinger operator on the half line II, J. Math. Phys. 55032103 (2014).

[9] R. Weder, Scattering theory for the matrix Schrödinger operator on the half line with general boundary conditions, J. Math. Phys. 56092103 (2015).

[10] V.S. Buslaev and L. D. Faddeev, formulas for traces for a singular Stürm-Liouville differential operator, Soviet Math. Doklady 1, 451-454 (1960).

[11] L. D. Faddeev, An expression for the trace of the difference between two singular differential operators of SturmLiouville type, Dokl. AN SSSR 115 N5, 878-881 (1957) ( russian).

[12] D. R. Yafaev, Mathematical Scattering Theory: Analytic Theory, Amer. Math. Soc. Providence, RI, 2010.

[13] N. I. Gerasimenko, The inverse scattering problem on a noncompact graph, Theoret. Math. Phys. 75, 460-470 (1988).

[14] N. I. Gerasimenko and B. S. Pavlov, A scattering problem on noncompact graphs, Theoret. Math. Phys. 74, 230-240 (1988).

[15] B. Gutkin and U. Smilansky, Can one hear the shape of a graph? J. Phys. A 34, 6061-6068 (2001).

[16] P. Kurasov and F. Stenberg, On the inverse scattering problem on branching graphs, J. Phys. A 35, 101-121 (2002).

[17] P. Kuchment, Quantum graphs. I. Some basic structures, Waves Random Media 14, S107-S128 (2004).

[18] J. Boman and P. Kurasov, Symmetries of quantum graphs and the inverse scattering problem, Adv. Appl. Math. 35, 58-70 (2005).

[19] P. Kuchment, Quantum graphs. II. Some spectral properties of quantum and combinatorial graphs, J. Phys. A 38, 4887-4900 (2005).

[20] P. Kurasov and M. Nowaczyk, Inverse spectral problem for quantum graphs, J. Phys. A 38, 4901-4915 (2005). 
[21] G. Berkolaiko, R. Carlson, S. A. Fulling, and P. Kuchment (eds.), Quantum Graphs and their Applications, Contemporary Mathematics, 415, Amer. Math. Soc., Providence, RI, 2006.

[22] P. Exner, J. P. Keating, P. Kuchment, T. Sunada, and A. Teplyaev (eds.), Analysis on graphs and its applications, Proc. Symposia in Pure Mathematics, 77, Amer. Math. Soc., Providence, RI, 2008.

[23] J. Behrndt and A. Luger, On the number of negative eigenvalues of the Laplacian on a metric graph, J. Phys. A 43, $474006(2010)$.

[24] P. Kurasov and M. Nowaczyk, Geometric properties of quantum graphs and vertex scattering matrices, Opuscula Mathematica 30, 295-309 (2010).

[25] G. Berkolaio and P. Kuchment, Introduction to Quantum Graphs. Mathematical Surveys and Monographs 186 Am. Math. Soc, Providence, R I 2013.

[26] Z. S. Agranovich and V. A. Marchenko, The Inverse Problem of Scattering Theory, Gordon and Breach, New York, 1963.

[27] A. Erdélyi, Asymptotic Expansions, Dover, New York, 1956. 